\title{
SENSITIVE ANALYSIS OF OBSERVATION MODEL FOR HUMAN TRACKING USING A STOCHASTIC PROCESS
}

\author{
W. Nakanishi ${ }^{\text {a, } *}$ and T. Fuse ${ }^{\text {a }}$ \\ ${ }^{a}$ Dept. of Civil Engineering, The University of Tokyo, 7-3-1 Hongo, Bunkyo, Tokyo 1138656, Japan \\ - (nakanishi, fuse)@civil.t.u-tokyo.ac.jp
}

KEY WORDS: Observations, Modelling, Tracking, Image, Analysis, Close Range

\begin{abstract}
:
This paper aims at obtaining basic knowledge about characteristics of observation models for human tracking method as a stochastic process. As human tracking in actual cases are complicated, we cannot always use the same observation models for every situation. Thus in most cases observation models are set empirically so far. In order to achieve an efficient choice of models and parameters, understanding some advantages and disadvantages of such models regarding to observation conditions is important. In this paper we conduct a sensitive analysis on some types of observation models. In particular, we obtain both colour and range information at a railway station. We prepare six predictive distributions as well as six models and parameters for both colour and range observation models. We calculate posterior distributions of each pattern, namely 36 patterns for both colour and range models. As a sensitive analysis we compare a value of a ground truth and an expected value of posteriors. We also compare variances of predictive and posterior distributions. Through this experimental results, we confirm our analysis method is efficient to obtain information about observation models. In fact, all models analysed are good in whole. One suggestive result is that colour models can deal with a predictive error in mean values, while range models in variances. Another is that under occlusions range models show a good performance.
\end{abstract}

\section{INTRODUCTION}

Human tracking method is classified roughly into two groups. One is that in each frame human candidates are detected and then the same person is identified in adjacent frames. Mathematically this problem can be solved as an optimization problem. The other is that, in each frame, each human position is predicted from that of previous frame and then accurate position is calculated by an observation model using observed information. As we can consider pedestrian behaviour model for successive frames, this method has an advantage to tracking many persons simultaneously in complex situations in which occlusions and proximities are occurred (e.g. Ali and Dailey, 2009; Nakanishi and Fuse, 2012). Mathematically this method should be solved as a stochastic process. Typical formulation for this method is based on Bayesian statistics: Predicted position is described by prior distribution, observed information corresponds to data and accurate position to posterior.

When we use this method, we define a likelihood function that describes likelihood of observed information occurred by given predicted position. We call this likelihood function "observation model" in human tracking method (explained later in detail). The main topic in this paper is how to set this observation model and its parameters. As both predicted positions and observations have uncertainty, it is difficult to choose which model to use. Thus, in actual case, most existing tracking methods set observation models just empirically and their performance and effect to tracking accuracy are rarely discussed. However, using empirical setting, we always have to set models and parameters manually. We cannot directly apply a setting on one observation site to another. Moreover, we might have to change model settings on the same observation site on different time or day.
Nevertheless according to uncertainty in observations, we cannot avoid to set some models and parameters adaptive to observation sites. However if we have some knowledge about advantages and disadvantages of each model to a certain observation condition, we can efficiently select observation models that seems to be adequate. Therefore in this paper we aim at obtaining basic knowledge about characteristics of observation models for human tracking method as a stochastic process. We obtain colour and range observations in a real situation. Next we set some predictive distributions and models and their parameters. Then we conduct a sensitive analysis on them as a basic experiment in order to achieve better modelling in the future. Through this experiment, we show our analysis method is efficient to obtain information about observation models. In the discussion, we shall present a difference between colour and range observation models revealed in this research.

The rest of the paper is organized as follows. In chapter 2, we briefly describe a stochastic human tracking process and in chapter 3 we propose a method to analyse observation models. Then in chapter 4 , we present settings for sensitive analysis, including settings of predictive distributions and observation models, as well as calculation techniques. In chapter 5, we apply this settings and conduct the sensitive analysis. We show the experimental results and some characteristics of each observation model. Finally we conclude the paper in chapter 6 .

\section{STOCHASTIC PROCESS MODELLING FOR HUMAN TRACKING}

\subsection{General State Space Model}

Human tracking can be regarded as a stochastic process. We use a general state space model (Figure 1) for this stochastic process modelling. 
In this modelling, we can estimate a dynamics of unobservable variables. A state vector $\mathbf{x}_{t}$ is a set of unobservable variables at time $t$ that we will estimate a true value. An observation vector $\mathbf{z}_{t}$ is a set of observable variables at time $t$. A system model $p\left(\mathbf{x}_{t} \mid \mathbf{x}_{t-1}\right)$ is a conditional density function that represents a dynamics of a state vector. An observation model $p\left(\mathbf{z}_{t} \mid \mathbf{x}_{t}\right)$ is also a conditional density function that represents a likelihood of realisation of $\mathbf{z}_{t}$ given $\mathbf{x}_{t}$.

After we obtain $\mathbf{z}_{1: t}=\left\{\mathbf{z}_{1}, \mathbf{z}_{2}, \ldots, \mathbf{z}_{t}\right\}$, a series of observations from time 1 to $t$, the posterior distribution of $\mathbf{x}_{t}$ is calculated by Bayes' theorem as follows:

$$
\begin{aligned}
& p\left(\mathbf{x}_{t} \mid \mathbf{z}_{1: t}\right) \propto p\left(\mathbf{z}_{t} \mid \mathbf{x}_{t}\right) p\left(\mathbf{x}_{t} \mid \mathbf{z}_{1: t-1}\right) \\
& \quad=p\left(\mathbf{z}_{t} \mid \mathbf{x}_{t}\right) \int p\left(\mathbf{x}_{t} \mid \mathbf{x}_{t-1}\right) p\left(\mathbf{x}_{t-1} \mid \mathbf{z}_{1: t-1}\right) d \mathbf{x}_{t-1}
\end{aligned}
$$

In this equation, $p\left(\mathbf{z}_{t} \mid \mathbf{x}_{t}\right)$ is an observation model, $p\left(\mathbf{x}_{t} \mid \mathbf{x}_{t-1}\right)$ is a system model and $p\left(\mathbf{x}_{t-1} \mid \mathbf{z}_{1: t-1}\right)$ is the estimation result at time $t-1$. If we need the very estimated value for $\mathbf{x}_{t}$, an expected value of $p\left(\mathbf{z}_{t} \mid \mathbf{x}_{t}\right)$ is usually used.

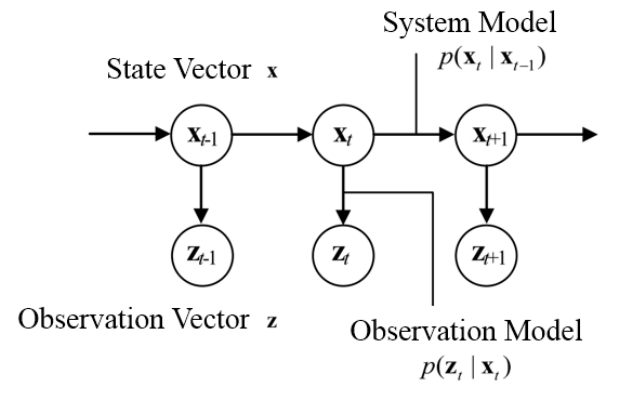

Figure 1. General state space model

\subsection{Stochastic Human Tracking Process}

We describe the stochastic human tracking process based on a general state space model.

We assume that a true value of a human position cannot be observed directly and represented as a conditional distribution. In each frame, a predictive distribution $p\left(\mathbf{z}_{t} \mid \mathbf{x}_{t-1}\right)$ as a prior distribution for a true value is calculated based on past observations. Then it is updated to a posterior distribution $p\left(\mathbf{z}_{t} \mid \mathbf{x}_{t}\right)$ after present observation data are acquired.

Formulation of system models are beyond the scope of this paper. There are some manners to set system models for human tracking. Some researches simply use a random walk model or a uniform motion model (e.g. Ali and Dailey, 2009). Others introduce a stochastic behaviour model such as a discrete choice model (e.g. Nakanishi and Fuse, 2012). In this paper, we assume that predictive distributions are given in advance by a certain system model.

We formulate observation models according to observations. For example, if we can use both colour and range information, we formulate models for each. Details are explained in the next chapter.

\section{METHOD OF SENSITIVE ANALYSIS}

As above, the role of an observation model is to reflect observation information to predictive distributions. However, a performance evaluation of observation models is difficult because of uncertainty in both predictive distributions and observation data. Thus existing tracking methods set observation models just empirically.

In this chapter we propose a method to analyse observation models. Firstly we set the components of a general state space model.

State Vector Settings: A state vector is defined as an ellipsoid and its coordinates that are human positions and shapes. In many researches, human shape is treated as an ellipse so this setting is considered to be natural. In this paper we fix four variables: three represent size of an ellipsoid and one represents height of a centroid of an ellipsoid. Thus we only consider two variables that represent a horizontal position of an ellipsoid.

Observation Vector Settings: An observation vector is defined as observations from stereo video camera, namely colour and range information. Thus we can discuss both colour and range observation models in this paper. At each observation point, we obtain three-dimensional colour information $(r, g$ and $b)$ and three-dimensional range information $(X, Y$ and $Z)$.

Predictive Distribution Settings: We set some types of predictive distributions regarding the accuracy of system models. Details are explained in the next chapter.

Observation Model Settings: We choose some observation models according to related works. Details are explained in the next chapter.

Ground Truth Data: We prepare a ground truth data manually in advance to evaluate posteriors. Details are explained in the next chapter.

The sensitive analysis in this paper is conducted on above settings as follows:

1. Make predictive distributions based on a ground truth.

2. Calculate posteriors on chosen observation models.

3. Compare some values: a ground truth and an expected value of a posterior (residuals); and variance of a predictive distribution and a posterior distribution.

\section{SETUP OF EXPERIMENTS}

In this section, we describe some settings for a sensitive analysis. Experimental results will be described in chapter 4 .

\subsection{Data Observation}

We take a video at the ticket gate of Tama-Plaza station, the railway station in a popular residential area about 20-kilometer west from central Tokyo. The video was taken from a site about 10-meter height, looking down obliquely (Figure 2). Both colour and range observations are acquired (Figure 3 ). 


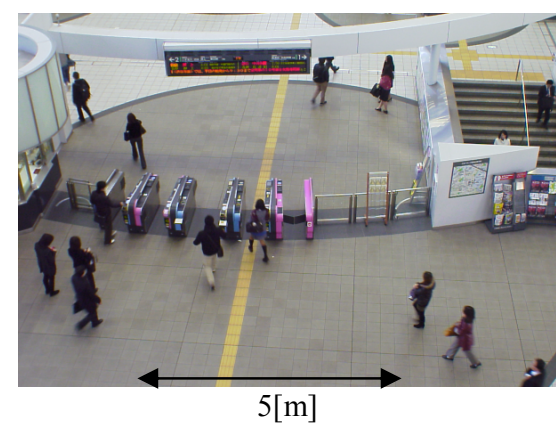

Figure 2. Observation site

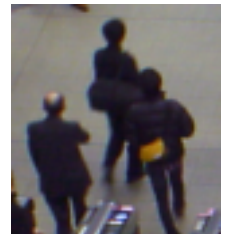

colour observations

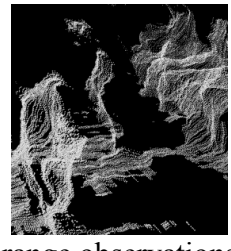

range observations

Figure 3. Example of colour and range observations

\subsection{Coordinate System and State Vector}

A coordinate system used in this paper is defined as shown in Figure 4. We assume that the ellipsoid of state vector stands vertically on the $x z$ plane. Thus the components of state vector is four constants ( $y$-value and size of ellipsoid) and two variables ( $x$ - and $z$-value). We set $y$-value to $1.0[\mathrm{~m}]$ and the size of ellipsoid to $1.6[\mathrm{~m}]$ height and $0.4[\mathrm{~m}]$ diameter.

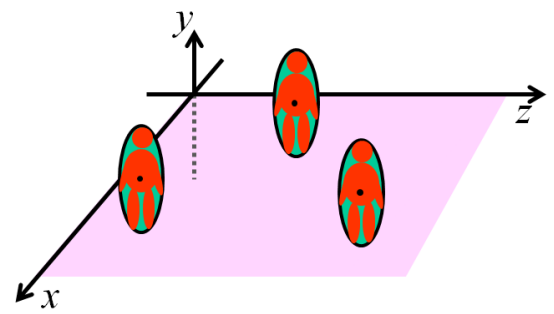

Ellipsoids stand vertically on $x z$ plane and their size and $y$-value of centroid are fixed.

Figure 4. Ellipsoid settings of human position and shape

\subsection{Ground Truth Dataset}

From this video we choose a few frames. Firstly we manually get passengers' positions from images (examples are shown in Figure 5). Then we label this dataset with its condition: normal or complicated (occluded and/or proximate). In Figure 5, blue ellipses show normal condition and red ones show complicated condition.

These conditions correspond to data accuracy. In normal conditions, observation information have relatively high reliability. On the other hand, in complicated conditions, observations don't have enough information to estimate accurate human positions. We check whether this difference may affect the performance of observation models, in the next chapter.

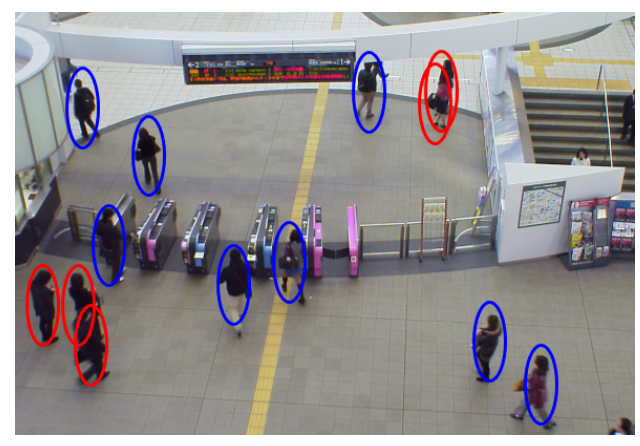

Figure 5. Example of the ground truth data

\subsection{Predictive Distributions}

We set predictive distributions shown below (Table 6 and Figure 7). In this experiment, we assume that all predictive distributions are two-dimensional isotropic normal distributions: $N\left(x^{*} t, \sigma^{2}\right) \times N\left(z * t, \sigma^{2}\right)$. Therefore they can be described as

$$
\begin{aligned}
p\left(\mathbf{x}_{t} \mid \mathbf{z}_{t-1}\right) & =\frac{1}{2 \pi \sigma^{2}} \exp \left[-\frac{\left(x_{*_{t}}-x_{t}\right)^{2}+\left(z_{*_{t}}-z_{t}\right)^{2}}{2 \sigma^{2}}\right] \\
& =\frac{1}{2 \pi \sigma^{2}} \exp \left[-\frac{d^{2}}{2 \sigma^{2}}\right]
\end{aligned}
$$

where $\quad \sigma^{2}=$ variance of normal distribution for each direction $x_{t}, z_{t}=$ predictive position on $x z$ plane at time $t$ $x * t, z *_{t}=$ average position on $x z$ plane at time $t$ $d=$ distance between predictive and average position

We omit the index $t$ in what follows, for we calculate any distributions at only time $t$ in this paper.

Each predictive distribution corresponds to a certain situation of system model accuracy. We set PD type 1-4 as average position equals to the ground truth with different variances, $2.5^{2}\left[\mathrm{~cm}^{2}\right]$, $5.0^{2}\left[\mathrm{~cm}^{2}\right], 7.5^{2}\left[\mathrm{~cm}^{2}\right]$ and $50.0^{2}\left[\mathrm{~cm}^{2}\right]$, respectively. This is a situation that system models can predict the expected value with high accuracy and these variances are assumed to correspond to confidence of prediction in various situations. We also set PD type 5-6 as average position are different from the ground truth with their distance $5.0[\mathrm{~cm}]$ and $10.0[\mathrm{~cm}]$ respectively, and with the same variance $5.0^{2}\left[\mathrm{~cm}^{2}\right]$. This is a situation that the prediction is not good because of some reasons. We also check whether this difference may affect the performance of observation models, in the next chapter.

Table.6 List of Predictive Distributions

\begin{tabular}{clc}
\hline PD type & Average position & Variance $\left[\mathrm{cm}^{2}\right]$ \\
\hline 1 & Ground truth & $2.5^{2}$ \\
\hline 2 & Ground truth & $5.0^{2}$ \\
\hline 3 & Ground truth & $7.5^{2}$ \\
\hline 4 & Ground truth & $50.0^{2}$ \\
\hline 5 & $\begin{array}{c}5.0[\mathrm{~cm}] \text { different } \\
\text { from ground truth }\end{array}$ & $5.0^{2}$ \\
\hline 6 & $\begin{array}{c}10.0[\mathrm{~cm}] \text { different } \\
\text { from ground truth }\end{array}$ & $5.0^{2}$ \\
\hline
\end{tabular}



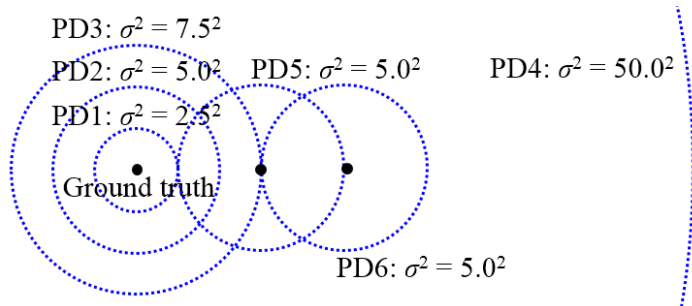

Blue lines illustrate an image of a standard deviation of each predictive distribution. Note that a probability that predicted position is within a distance of one sigma is about $47 \%(68 \% \times 68 \%)$.

Figure 7. Image of predictive distribution

\subsection{Observation Models}

We set some models to conduct a sensitive analysis. Because observation models are required to return likelihood of any position if human can be at that point, they should be continuous function, as predictive distribution is continuous. From this aspect, referring to previous research, we select models we employ in this paper.

Colour Observation Models: For colour observation models, we use Bhattacharyya coefficient

$$
p_{\text {colour }}(\mathbf{z} \mid \mathbf{x})=f(B)=f\left(\sum_{m} \sqrt{p_{m} q_{m}}\right)
$$

where $\quad B=$ Bhattacharyya coefficient

$m=$ pixel value

$p, q=$ normalized histogram of predictive position and a ground truth respectively

$p_{m}=$ relative frequency of pixel value $m$ in histogram $p$

$f=$ function set later

This is a coefficient correlation of colour histograms. Bhattacharyya coefficient compares similarity of colour histograms of a predicted position and a ground truth.

We employ two manners to generate histograms. A histogram is generated as follows: At predicted or ground truth position we make an ellipsoid representing human shape, and then make histogram of observed points inside that ellipsoid. Here we employ two manners to treat each pixel. One is treating every pixel equally (Uniform type) and the other is weighting pixels according to a distance from the centroid of the ellipsoid (Weighted type).

We calculate $B$ for each colour observed, $r, g$ and $b$ and then calculate product of them. We also employ three functions for $f(B)$ in equation (3). These are, $p_{\text {colour }}=B$ (linear model $), p_{\text {colour }}$ $=\exp (-5(1-B))($ Exp-5 model $)$ and $p_{\text {colour }}=\exp (-10(1-B))($ Exp10 model). The difference between each model is shown in Figure 8. In a qualitative manner, Exp-10 model is more sensitive to a difference between the histogram of a predictive position and a ground truth.

Thus at last we have six models for colour observations.

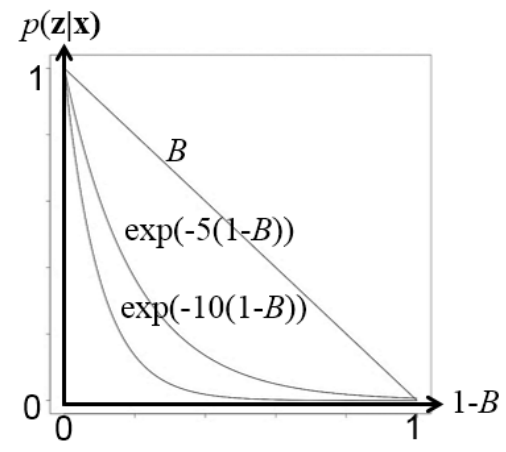

Figure 8. Likelihood of each colour model

Range Observation Models: For range observation models, we use a distribution according to a similarity between a shape of predicted ellipsoid and observed 3D points in actuality. For each pixel $P$ included in the ellipsoid, let $d(P)$ the distance from observed coordinates to the centre of the ellipsoid $O$. Let $P^{\prime}$ the point that a half line from $O$ to $P$ intersects the ellipsoid, and $\hat{d}(P)$ the distance from $O$ to $P^{\prime}$. We consider two model types as follows: Equation (4) measures a distance (Distance Type) and (5) a ratio (Ratio Type).

$$
\begin{aligned}
& p_{\text {range }}(\mathbf{z} \mid \mathbf{x})=1-\frac{1}{I} \sum_{p}\left(\left|d_{p}-\hat{d}_{p}\right|\right)^{\lambda} \\
& p_{\text {range }}(\mathbf{z} \mid \mathbf{x})=\frac{1}{I} \sum_{p}\left(\frac{d_{p}}{\hat{d}_{p}}\right)^{\lambda}
\end{aligned}
$$

where $\quad I=$ number of pixel $P$ in total

$\lambda=$ parameter set just below

We employ three values for $\lambda$ in Equation (4) and (5). These are $0.5,1.0$ and 2.0. Thus we also have six models for range observations.

\subsection{Calculation Techniques}

We use particle filter (Gordon, et al., 1993; Kitagawa, 1996) for calculating equation (1), for observation models are non-linear. In this work we use 1024 particles to approximate posteriors. We also use Halton sequences (Halton, 1964) to generate prior (predictive) distributions. They are typical quasi-random number sequences. Thus good approximation of posteriors with less number of particles is expected.

\section{EXPERIMENTAL RESULTS}

In this section, we describe experimental results.

\subsection{Results}

As described in chapter 3, we calculate residuals and variances of each posterior as a sensitive analysis. Table 9 and 10 show residuals and variances in each observation model, parameter setting and predictive distribution. 
Table 9. Sensitive analysis on colour models

\begin{tabular}{|c|c|c|c|c|c|c|c|c|c|}
\hline \multirow[t]{2}{*}{$\begin{array}{l}\text { Histogram } \\
\text { type }\end{array}$} & \multirow[t]{2}{*}{$\begin{array}{l}\text { PD } \\
\text { Type }\end{array}$} & \multirow[t]{2}{*}{$\begin{array}{l}\text { Model } \\
\text { type }\end{array}$} & Residual [cm] & $\begin{array}{l}\text { Variance of } \\
\text { posterior } \\
\text { distribution }\left[\mathrm{cm}^{2}\right]\end{array}$ & \multirow[t]{2}{*}{\begin{tabular}{|l} 
Model \\
type
\end{tabular}} & \multirow[t]{2}{*}{$\begin{array}{l}\text { PD } \\
\text { Type }\end{array}$} & \multirow[t]{2}{*}{$\begin{array}{l}\text { Parameter } \\
\text { setting }\end{array}$} & \multirow{2}{*}{$\begin{array}{l}\text { Residual }[\mathrm{cm}] \\
\\
\text { Dataset: } \\
\text { Normal / } \\
\text { Complicated / } \\
\text { All }\end{array}$} & \multirow{2}{*}{$\begin{array}{l}\begin{array}{l}\text { Variance of } \\
\text { posterior } \\
\text { distribution } \\
{\left[\mathrm{cm}^{2}\right]}\end{array} \\
\text { Dataset: } \\
\text { Normal / } \\
\text { Complicated / } \\
\text { All }\end{array}$} \\
\hline & & & $\begin{array}{l}\text { Dataset: } \\
\text { Normal / } \\
\text { Complicated / } \\
\text { All }\end{array}$ & $\begin{array}{l}\text { Dataset: } \\
\text { Normal / } \\
\text { Complicated / } \\
\text { All }\end{array}$ & & & & & \\
\hline \multirow{18}{*}{ Uniform } & \multirow{3}{*}{1} & Linear & $0.3 / 0.0 / 0.2$ & $7.5 / 8.4 / 7.8$ & \multirow{18}{*}{ Distance } & \multirow{3}{*}{1} & $\lambda=0.5$ & $1.3 / 0.0 / 1.0$ & $7.6 / 8.8 / 7.9$ \\
\hline & & Exp-5 & $0.5 / 0.2 / 0.4$ & $5.7 / 40.0 / 17.1$ & & & $\lambda=1.0$ & $1.3 / 0.0 / 0.9$ & $7.6 / 8.8 / 7.9$ \\
\hline & & Exp-10 & $0.5 / 0.3 / 0.4$ & $4.1 / 6.4 / 4.9$ & & & $\lambda=2.0$ & $1.3 / 0.0 / 0.9$ & $7.6 / 8.8 / 7.9$ \\
\hline & \multirow{3}{*}{2} & Linear & $1.3 / 0.3 / 1.0$ & 25.4 / $86.1 / 45.6$ & & \multirow{3}{*}{2} & $\lambda=0.5$ & $1.8 / 0.1 / 1.3$ & $30.4 / 35.2 / 31.8$ \\
\hline & & Exp-5 & $1.5 / 1.2 / 1.4$ & $17.9 / 587 / 208$ & & & $\lambda=1.0$ & $1.8 / 0.0 / 1.3$ & $30.5 / 35.1 / 31.8$ \\
\hline & & Exp-10 & $1.4 / 0.8 / 1.2$ & $11.0 / 25.5 / 15.9$ & & & $\lambda=2.0$ & $1.8 / 0.0 / 1.3$ & $30.5 / 35.1 / 31.8$ \\
\hline & \multirow{3}{*}{3} & Linear & $2.7 / 1.3 / 2.2$ & 49.7 / 679 / 260 & & \multirow{3}{*}{3} & $\lambda=0.5$ & $2.5 / 0.1 / 1.8$ & $66.9 / 79.2 / 70.4$ \\
\hline & & Exp-5 & $2.7 / 3.1 / 2.8$ & $34.1 / 1716 / 595$ & & & $\lambda=1.0$ & $2.6 / 0.1 / 1.9$ & $67.2 / 78.8 / 70.5$ \\
\hline & & Exp-10 & $2.2 / 1.3 / 1.9$ & $18.6 / 59.6 / 32.3$ & & & $\lambda=2.0$ & $2.7 / 0.0 / 1.9$ & $67.3 / 78.7 / 70.5$ \\
\hline & \multirow{3}{*}{4} & Linear & $12.8 / 36.9 / 20.8$ & $2140 / 15069 / 6450$ & & \multirow{3}{*}{4} & $\lambda=0.5$ & $19.8 / 20.3 / 19.9$ & $2110 / 2093 / 2104$ \\
\hline & & Exp-5 & $44.1 / 60.2 / 49.5$ & $21065 / 36356 / 26162$ & & & $\lambda=1.0$ & $19.9 / 21.0 / 20.2$ & $2116 / 2081 / 2106$ \\
\hline & & Exp-10 & $7.6 / 6.2 / 7.1$ & $1619 / 2083 / 1774$ & & & $\lambda=2.0$ & $19.9 / 21.3 / 20.3$ & $2119 / 2076 / 2106$ \\
\hline & \multirow{3}{*}{5} & Linear & $3.9 / 5.0 / 4.3$ & $26.8 / 804 / 286$ & & \multirow{3}{*}{5} & $\lambda=0.5$ & $5.8 / 5.1 / 5.6$ & $31.1 / 35.3 / 32.3$ \\
\hline & & Exp-5 & $2.9 / 6.0 / 3.9$ & $20.5 / 2148 / 730$ & & & $\lambda=1.0$ & $5.9 / 5.1 / 5.6$ & $31.1 / 35.2 / 32.3$ \\
\hline & & Exp-10 & $1.8 / 2.8 / 2.1$ & $13.0 / 51.4 / 25.8$ & & & $\lambda=2.0$ & $5.9 / 5.0 / 5.6$ & $31.1 / 35.1 / 32.3$ \\
\hline & \multirow{3}{*}{6} & Linear & $7.8 / 16.0 / 10.5$ & 29.0 / 6617 / 2225 & & \multirow{3}{*}{6} & $\lambda=0.5$ & $10.2 / 10.1 / 10.1$ & $31.5 / 34.8 / 32.5$ \\
\hline & & Exp-5 & $6.2 / 18.2 / 10.2$ & $26.7 / 8841 / 2965$ & & & $\lambda=1.0$ & $10.2 / 10.0 / 10.2$ & $31.5 / 34.7 / 32.4$ \\
\hline & & Exp-10 & $4.0 / 6.0 / 4.6$ & $19.1 / 253 / 97.0$ & & & $\lambda=2.0$ & $10.2 / 10.0 / 10.2$ & $31.5 / 34.6 / 32.4$ \\
\hline \multirow{18}{*}{ Weighted } & \multirow{3}{*}{1} & Linear & $0.2 / 0.0 / 0.2$ & $7.6 / 8.5 / 7.9$ & & \multirow{3}{*}{1} & $\lambda=0.5$ & $1.3 / 0.0 / 1.0$ & $7.6 / 8.8 / 7.9$ \\
\hline & & Exp-5 & $0.4 / 0.1 / 0.3$ & $5.7 / 32.5 / 14.7$ & & & $\lambda=1.0$ & $1.4 / 0.0 / 1.0$ & $7.6 / 8.8 / 7.9$ \\
\hline & & Exp-10 & $0.4 / 0.1 / 0.3$ & $4.4 / 7.1 / 5.3$ & & & $\lambda=2.0$ & $1.4 / 0.1 / 1.0$ & $7.6 / 8.8 / 7.9$ \\
\hline & & Linear & $1.2 / 0.2 / 0.9$ & $24.3 / 85.0 / 44.5$ & & & $\lambda=0.5$ & $1.8 / 0.1 / 1.3$ & $30.4 / 35.4 / 31.8$ \\
\hline & 2 & Exp-5 & $1.3 / 0.8 / 1.1$ & $15.9 / 442 / 158$ & & 2 & $\lambda=1.0$ & $1.9 / 0.1 / 1.4$ & $30.3 / 35.6 / 31.8$ \\
\hline & & Exp-10 & $1.2 / 0.5 / 0.9$ & $9.9 / 24.1 / 14.6$ & & & $\lambda=2.0$ & $2.0 / 0.2 / 1.5$ & $30.2 / 35.8 / 31.8$ \\
\hline & & Linear & $2.5 / 0.8 / 1.9$ & $45.6 / 424 / 172$ & & & $\lambda=0.5$ & $2.5 / 0.1 / 1.8$ & $66.8 / 79.7 / 70.5$ \\
\hline & 3 & Exp-5 & $2.3 / 2.2 / 2.2$ & 28.4 / 1284 / 447 & & 3 & $\lambda=1.0$ & $2.4 / 0.2 / 1.8$ & $66.4 / 80.7 / 70.5$ \\
\hline & & Exp-10 & $1.8 / 0.9 / 1.5$ & $14.7 / 48.0 / 25.8$ & & & $\lambda=2.0$ & $2.5 / 0.4 / 1.9$ & $65.8 / 82.1 / 70.5$ \\
\hline & & Linear & $12.8 / 28.5 / 18.0$ & $2000 / 10056 / 4685$ & & & $\lambda=0.5$ & $19.7 / 20.4 / 19.9$ & $2114 / 2098 / 2110$ \\
\hline & 4 & Exp-5 & $36.7 / 53.3 / 42.2$ & $17527 / 33646 / 22900$ & & 4 & $\lambda=1.0$ & $19.6 / 19.6 / 19.6$ & $2111 / 2117 / 2113$ \\
\hline & & Exp-10 & $4.5 / 3.5 / 4.2$ & $580 / 1160 / 773$ & & & $\lambda=2.0$ & $19.5 / 18.4 / 19.1$ & $2106 / 2151 / 2119$ \\
\hline & & Linear & $3.7 / 4.7 / 4.0$ & $25.7 / 550 / 200$ & & & $\lambda=0.5$ & $5.8 / 5.1 / 5.6$ & $31.0 / 35.3 / 32.2$ \\
\hline & 5 & Exp-5 & $2.4 / 5.2 / 3.3$ & $17.8 / 1693 / 576$ & & 5 & $\lambda=1.0$ & $5.8 / 5.2 / 5.6$ & $30.9 / 35.5 / 32.2$ \\
\hline & & Exp-10 & $1.4 / 2.9 / 1.9$ & 10.4 / 38.9 / 19.9 & & & $\lambda=2.0$ & $5.7 / 5.3 / 5.6$ & $30.7 / 35.8 / 32.2$ \\
\hline & & Linear & $7.3 / 13.1 / 9.3$ & $27.8 / 4669 / 1575$ & & & $\lambda=0.5$ & $10.2 / 10.1 / 10.1$ & $31.5 / 34.7 / 32.4$ \\
\hline & 6 & Exp-5 & $5.4 / 16.0 / 8.9$ & $24.3 / 7636 / 2562$ & & 6 & $\lambda=1.0$ & $10.1 / 10.2 / 10.1$ & $31.4 / 34.7 / 32.4$ \\
\hline & & Exp-10 & $2.9 / 5.9 / 3.9$ & $14.2 / 157 / 61.7$ & & & $\lambda=2.0$ & $10.0 / 10.4 / 10.1$ & $31.4 / 34.8 / 32.3$ \\
\hline
\end{tabular}




\subsection{Discussions}

All models were good as a whole in terms of residuals and variances in predictive distribution type 1-3. As PD type 1-3 are considered to be good prediction accuracy, this tendency is the most important for observation models. There are little difference between histogram type for colour and model type for range models. So from another aspect, considering computational speed, uniform histogram with linear model for colour and Ratio model with $\lambda=2.0$ for range model are good. This is because some variables in that models are already calculated when being evaluated whether the point is inside the ellipsoid or not.

The results on PD type 5 and 6 show that colour models can deal with a predictive error in expected values especially in normal conditions. It is natural to think that we can distinguish the human and other objects by the difference in colour information. On the other hand, these models cannot well deal with a predictive error in variances except Exp-10 models. We can understand this tendency like this: Colour models can judge whether an object is a certain human or not, but cannot search the very position of it.

The results on PD type 4 show that range models can deal with a predictive error in variances, for posteriors' variances are smaller than that of prior (2500). Range models are also good at dealing with complicated situations, especially with occlusions.

Exp-10 colour models show different behaviours from others. They seem to achieve decreasing variances as well as small residuals. However when we use Exp-10 models, fairly accurate system models are required. If a predictive distribution is far from a true value, then that models cannot return any values, as illustrated in Figure 8. In case we have confidence on system models, we can use Exp-10 models and expect a good performance.

Through this analysis we showed the way to evaluate observation models. Only simple comparisons of residuals and variances can provide us some valuable information. So the next step is to evaluate other models and analyse many tendencies around observation models and observation data. In addition, a method to compare distributions directly could provide more information. Moreover, a joint analysis with system models is expected.

\section{CONCLUSION}

In this paper we conducted the sensitive analysis on observation models for human tracking methods as a stochastic process. We developed the method to evaluate and compare some observation models. Through experiments we confirm that our method is efficient. Results showed some suggestive characteristics of models: All models analysed are good in whole; Colour models can deal with a predictive error in mean values, while range models in variances; Under occlusions range models show a good performance.

Future works will firstly aim to improve proposed method through conducting analyses on other data and models, as well as developing a method to compare distributions directly. Although an adequate observation model may depend on situations of observation site, we believe that analyses in many cases can provide us much knowledge. Based on that knowledge we can use some observation models simultaneously and dynamically select the best one according to accuracy of predictive distribution and data acquisition. We will also expand this method to a joint analysis with system models like identical twin experiments in the field of data assimilation. These experiments will lead us to a new standpoint of desirable system models, according to accuracy of observation data and observation model.

\section{References:}

Ali, I. and Dailey, M., 2009. Multiple human tracking in highdensity crowds, Advanced Concepts for Intelligent Vision Systems, Vol. LNCS 5807, pp.540-549.

Gordon, N. J., Salmond, D. J. and Smith, A. F. M., 1993. Novel Approach to Nonlinear / Non-Gaussian Bayesian State Estimation. Radar and Signal Processing, IEE Proceedings F, 140(2), pp.107-113.

Halton, J. H., 1964. Algorithm 247: Radical-inverse quasirandom point sequence, Communications of the $A C M, 7(12)$, pp.701-702,

Kitagawa, G., 1996. Monte Carlo Filter and Smoother for NonGaussian Nonlinear State Space Models. Journal of Computational and Graphical Statistics, 5(1), pp.1-25.

Nakanishi, W. and Fuse, T., 2012. Multiple Human Tracking in Complex Situation by Data Assimilation with Pedestrian Behavior Model. ISPRS Archives, 39-B3, pp.409-414.

\section{ACKNOWLEDGEMENTS:}

A part of this research was supported by Japan Society for the Promotion of Science, Grant-in-Aid for JSPS Fellows (25 9044). 